\title{
Empleo de la Historia del Arte para la adquisición de nociones estructurantes del área de Conocimiento del Entorno en Educación Infantil: espacio y tiempo
}

\section{Employment of the History of the Art for the acquisition of structuring notions on the subject Environmental Knowledge in Infantile Education: space and time}

\author{
Ana María Mendioroz-LaCAmbra \\ Universidad Pública de Navarra: Facultad de Ciencias Humanas y Sociales. \\ Dpto. Geografía e Historia. \\ anamaria.mendioroz@unavarra.es
}

Recibido: 16 de mayo de 2012

Aprobado: 24 de julio de 2012

\begin{abstract}
Resumen
Presentamos una experiencia llevada a cabo por alumnas de $3^{\circ}$ curso del Grado de Maestro de Educación Infantil en la UPNA, durante las prácticas escolares. La adquisición de los constructos espacio-temporales en Educación Infantil, imprescindibles para estructurar el pensamiento, presenta no pocas dificultades; esto es debido a la naturaleza de los aprendizajes, tanto como a las características psicológicas de esta etapa. Para facilitar su adquisición de forma significativa, y debido a que su carácter polisémico le aporta un alto valor educativo, proponemos el recurso de la imagen. En este caso concreto presentamos el proyecto realizado en segundo ciclo de educación Infantil, sobre la base de una obra de arte pictórica: La familia del Duque de Osuna, pintada por Goya en 1788.
\end{abstract}

Palabras Clave: obra de arte, espacio, tiempo.

Mendioroz-Lacambra, A. M. (2013): Empleo de la Historia del Arte para la adquisición de nociones estructurantes del área de Conocimiento del Entorno en Educación Infantil: espacio y tiempo. Arte, Individuo y Sociedad, 25(3) 392-405

\begin{abstract}
We present the experience that was carried out by students of 3rd course of Teaching Education of UPNA during their training period in primary schools. The acquisition of constructions of spacetime necessary to achieve structured thinking in primary education, is a very demanding task due to learning process as well as to psychological condition of children of this age. With the aim to support a meaningful acquisition, we propose the use of image, bearing in mind that its polysemic nature adds an extremely high educational value,. In this particular case, the project carried out was based in the use of pictorial art in the second cycle of Infantile Education: The family of the Duke of Osuna, painted by Goya in 1788.
\end{abstract}

Mendioroz-Lacambra, A. M. (2013): Employment of the History of the Art for the acquisition of structuring notions on the subject Environmental Knowledge in Infantile Education: space and time. Arte, Individuo y Sociedad, 25(3) 392-405 
Sumario: 1. Introducción, 2. Metodología, 2.1. Primera Fase, 2.2. Segunda Fase, 2.3. Tercera fase, 3. Resultados, 4. Conclusiones. Referencias.

\section{Introducción}

El carácter globalizador que caracteriza a la etapa de Educación Infantil, exige que el estudio del entorno sea planteado desde la complementariedad de las tres áreas en las que se estructura el currículo (Bassedas, Huguet y Olé, 2002), aunque la mayor parte de contenidos propios de las ciencias sociales, se incluyan en el área de Conocimiento del Entorno (Cuenca, 2006).

Se entiende por entorno los factores naturales, sociales, culturales y psicológicos que inciden en el comportamiento y que dotan de significación las actuaciones, a partir de la observación y la experimentación; de ahí que los contenidos referentes a la formación del pensamiento social, al conocimiento de los fenómenos, sucesos, productos sociales y culturales, así como a la identificación de algunos cambios en el modo de vida en relación con el paso del tiempo, procedan de la percepción global de lo social, lo espacial y lo temporal (Aranda, 2003), ejes vertebradores propios de las ciencias sociales y del área.

La adquisición de los constructos espacio temporales, son imprescindibles para estructurar el pensamiento, pero presentan grandes dificultades, tanto por la naturaleza de los aprendizajes como por las características psicológicas de esta etapa (Sánchez, 2000). Para facilitar su dominio de forma significativa, proponemos, siguiendo a Arque i Bertran, (2002), el recurso de la imagen y del patrimonio artístico en general, como proponen entre otros Estepa, Wamba, y Jiménez, (2004), Bartolomeis, (2006), Ávila, (2003), Ballesteros, (2003), Bardavio y González, (2003), Benites y Fitchner, (2004), Calaf, (2010, 2011), Cruz Solís, (2006), Gavaldá, (2003), González Monfort, (2008), Huerta, (2011), Medina, (2011) o Rielo, (2010), ya que debido a su carácter polisémico tiene un alto valor educativo.

De hecho, facilita actividades de carácter integrador y transversal, que permiten entender y valorar el entorno de forma holística (Hernández Cardona, 2004, 2011); cuenta historias que se desarrollan en el espacio y el tiempo (Cuenca y Domínguez, 2000); despierta la imaginación, la empatía para comprender el pasado y explicarlo desde puntos de vista distintos (Cuenca y Estepa, 2005); desarrolla habilidades cognitivas que permiten formular hipótesis, realizar análisis y síntesis, potenciando el pensamiento de más alto nivel (Calaf y Fontal, 2004). Contribuye en suma, y de una forma muy transparente, a reconstruir el pasado, a comprender los cambios, continuidades y simultaneidades con explicaciones multicausales, y evita la recepción del conocimiento de forma memorística e inamovible (Trepat, 2005). Además, ayuda a entender el presente y a construir pensamiento y explicación histórica, empleando el método histórico, tal y como lo hacen los historiadores. Su empleo, favorece la construcción de los principios de espacialidad y temporalidad, básicos para comprender el entorno y tomar conciencia de que el presente tiene que ver con el pasado y avanza hacia el futuro (Zabala, 2006). Finalmente, contribuye a trabajar las competencias básicas, que deberán ser adquiridas durante las siguientes etapas educativas. 
Para lograrlo, es imprescindible el empleo de una metodología derivada de la propia disciplina (Socias, 1996). Partiendo de núcleos temáticos significativos y centrada en la forma de ver, debe integrar contenidos conceptuales, procedimentales y actitudinales; debe permitir el desarrollo de varias lecturas, tanto desde el punto de vista connotativo, como denotativo y formal, facilitando una perspectiva que presente el contexto y el pensamiento, que posibilite la relación entre expresión y significación del objeto, a través de los elementos que ordenan el pensamiento visual, para desentrañar finalmente el significado de la obra. En suma, un método que integre el análisis formal, iconográfico y sociológico, teniendo en cuenta que la observación se produce desde la actualidad.

Esta experiencia se llevó a cabo en la asignatura de Didáctica del Entorno Social, con estudiantes de tercer curso del grado de Maestro de Educación Infantil en la UPNA, en el marco del prácticum. La intervención consistió en analizar los resultados de aprendizaje sobre las nociones estructurantes clave del área, empleando como recurso de aprendizaje la Historia del Arte. Desde un marco cognitivo/constructivista, y partiendo de los conocimientos previos, se realizó una práctica guiada por la teoría y la investigación. Se presentó la materia de acuerdo a la lógica de la ciencia y a la madurez de la etapa, y se emplearon materiales conceptualmente transparentes, intentando que el grupo compartiera significados e integrara el nuevo conocimiento de forma sustantiva, buscando la significatividad de los aprendizajes. No en vano, el conocimiento que exige la sociedad actual, no pasa por memorizar datos, entre otras cosas por la imposibilidad de procesarlos, sino en conocer los procesos para llegar a la información, analizarla y conceptualizarla de manera personal y crítica. Por tanto el conocimiento no es sólo erudición, sino la habilidad de crearlo y transferirlo.

La reforma educativa pretende responder a este reto, y como docentes tenemos la responsabilidad de que nuestro alumnado, futuro profesorado, sea competente para crear escenarios educativos ricos, que faciliten al alumnado de Educación Infantil conocer, interpretar y participar de forma autónoma y responsable en el entorno. En las aulas de esta etapa, hay que trabajar en la misma dirección que en el resto de los espacios educativos; por ello es necesario iniciar el desarrollo de las competencias básicas, que como la de aprender a aprender, se irán consolidando a lo largo de las siguientes etapas educativas.

\section{Metodología}

El proyecto se aplicó en dos aulas del segundo ciclo de Educación Infantil durante cinco sesiones, en el marco de una metodología cualitativa ligada al paradigma interpretativo, de creciente utilización en el campo de la investigación en educación (Ruiz, 1996), ya que permite analizar la realidad educativa en su globalidad, siguiendo un proceso inductivo. Para facilitar la recogida de datos, se empleó el diario de campo donde se registraron las observaciones realizadas, así como grabaciones audiovisuales, que facilitaron el tratamiento y la gestión de la información.

Se partió de la hipótesis de que el empleo de fuentes históricas de tipo iconográfico, ayuda al alumnado de Educación Infantil a elaborar los constructos espacio-temporales y culturales, de manera significativa. Los objetivos propuestos fueron: desarrollar 
nociones básicas espaciales y temporales a través de la lectura de la imagen; reconocer alguna característica cultural mediante la observación, manipulación, interpretación y análisis de la imagen, así como comprender y explicar de forma causal la sucesión, el cambio y la permanencia a través del tiempo, iniciando a los niños en el método histórico y en el empleo de fuentes primarias, para comprender el presente.

Se optó por el empleo de una metodología activa, indagativa, de tal forma que el alumnado fuera el auténtico protagonista de su aprendizaje, partiendo de sus intereses, a través de la observación, la manipulación, la comparación y la comunicación. Se diseñaron diferentes proyectos, todos ellos en base a fuentes primarias de carácter icónico. En esta ocasión, nos centraremos en el elaborado a partir del cuadro que Goya pintó en 1788, La familia del duque de Osuna. La experiencia llevada a cabo por las alumnas, incluyó las siguientes fases.

\subsection{Primera Fase}

Se presentó el problema: ¿Cómo se puede facilitar en el aula de Educación Infantil la adquisición de los constructos espacio temporales?, y se constituyeron los grupos de trabajo, integrados cada uno de ellos por cinco alumnas. Se les entregó bibliografía sobre estos contenidos, para que los analizaran desde el marco científico y psicopedagógico, y conocieran el estado actual de las investigaciones. Una vez que pusieron en valor la importancia que tienen en la estructuración del pensamiento, así como la dificultad que presenta su consecución, se les pidió que analizaran su tratamiento en el currículo de la etapa, tanto en el área de Conocimiento del Entorno, como en el resto de las áreas, a nivel de contenidos, objetivos y criterios de evaluación. Descubrieron de esta forma las posibilidades de convertir el currículo en trabajo de aula, y valoraron la presencia de estos constructos a nivel curricular llegando a la conclusión de que no era todo lo explícita que sería conveniente, quizás por las limitaciones defendidas en las teorías psicopedagógicas de corte clásico.

\subsection{Segunda Fase}

Para conocer qué actitudes habían desarrollado nuestras alumnas sobre contenidos artísticos, y su posible utilización como recurso instruccional en el proceso educativo, les pasamos una encuesta. Muchas de ellas provenían de los ciclos superiores de Formación Profesional, y los conocimientos sobre historia del arte eran muy limitados. Las que habían estudiado la materia en Bachillerato, tenían mayor bagaje de contenidos pero con una concepción de la materia muy positivista. Pudimos comprobar que en ninguno de los casos, eran conscientes de su potencial educativo. Para subsanarlo, se les entregó bibliografía que les permitió reflexionar sobre los procesos y mecanismos implicados en la creación, así como en su valor formativo. De esta manera pusieron en valor el papel que juega el arte en el desarrollo del pensamiento. Por una parte, y desde un nivel afectivo, referente a los sentimientos, sirve entre otras cosas para reforzar la conciencia de pertenencia a una colectividad, favoreciendo la multiculturalidad. Desde otro puramente sensorial, de percepción, actúa como base necesaria de todo conocimiento; y finalmente desde el cognitivo, potencia la observación, descripción, argumentación y conceptualización o reflexión, 
todos ellos procesos mentales que permiten desarrollar estrategias de pensamiento del más alto nivel (Eisner, 2001).

Además fueron capaces de valorar el empleo de la obra de arte como fuente de conocimiento histórico (Cooper, 2002), ya que facilita la formulación de hipótesis, la realización de análisis y síntesis, así como el desarrollo de la abstracción y la imaginación, necesarias para sopesar diferentes versiones de una misma historia, entender las causas que la motivaron y sus efectos, así como el contexto en el que fue creada; en suma, los valores y mensajes mediados por las imágenes. Finalmente, reconocieron a la imagen su valor para aprender a ver, vivir y pensar el espacio, así como para hacer transparentes y explicar de forma causal, los conceptos de sucesión, cambio y continuidad, es decir llenar de contenido el tiempo, también el histórico(Candau, 2006).

\subsection{Tercera Fase}

Desde un marco teórico sólido que les permitió fundamentar su trabajo tanto a nivel psicopedagógico, como disciplinar, curricular y didáctico, procedieron al diseño del proyecto de intervención para el aula de 5 años, basado en el empleo del cuadro pintado por Goya en 1788, La Familia del Duque de Osuna (Fig. 1).

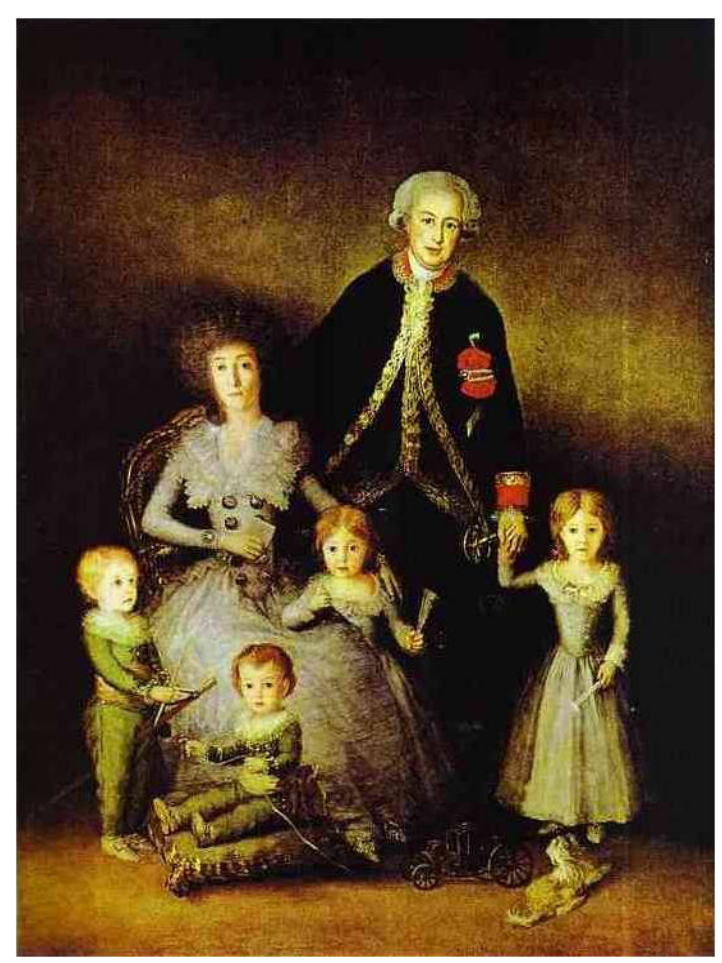

Figura 1. La Familia del Duque de Osuna, 1788. (Goya). 
Eligieron esta obra libremente, porque, entre otras cosas, les permitía trabajar contenidos culturales significativos para los niños como la ropa, el peinado, los juguetes o el tipo de familia; $y$, además, posibilitaba las comparaciones con la época actual, a partir de la observación. Partieron de los siguientes objetivos: que los alumnos fueran capaces de desarrollar nociones básicas de orientación y posición relativa de los objetos, teniendo como referencia hitos externos al propio niño ; que fueran capaces de afianzar conceptos temporales referentes a la orientación (pasado, presente, futuro), sucesión, continuidad y cambio por el paso del tiempo, así como formular conjeturas sobre causas y consecuencias, en base a referencias de contenidos culturales presentes en la ilustración, que les permitieran percibir algunas de las características propias de la época y su evolución en la actualidad, todo ello mediante la observación, comparación, análisis e interpretación de la imagen. Para conseguirlo, planificaron y diseñaron una serie de actividades encaminadas a trabajar las diferentes lecturas: connotativa, denotativa y formal (Juanola y Calvo 2006).

Como la intervención debía ser corta, cinco sesiones de 35 minutos, de todas las tareas que habían planificado, eligieron una actividad para cada día, de tal forma que el resto fueran trabajadas por las tutoras del centro una vez finalizado el prácticum, a modo de refuerzo y ampliación. La primera debía servirles de motivación y permitirles evidenciar el grado de dominio alcanzado por los niños sobre orientación espacial y temporal. Estimaron oportuno trabajar la lectura connotativa de forma colaborativa con una actividad grupal y sin embargo optaron por realizar la denotativa en pequeños grupos, con dos actividades que sirvieran a su vez como evaluadoras de los aprendizajes. Finalmente, una más para trabajar la lectura formal, que por la falta de tiempo debía servirles también para consolidar los constructos espaciales.

Tras la actividad inicial, se realizó la lectura connotativa en contacto directo con la obra. Se planteó como aproximación guiada, buscando el desarrollo de la afectividad, la imaginación y la empatía. Partiendo de una primera observación libre, se les guió con un cuestionario de preguntas abiertas como las siguientes: qué ves, quiénes son, a qué huele, de qué hablan, por qué están ahí, imagínate dentro, cuéntame su historia, qué es lo que más te gusta, qué quieres saber... que les ayudaron a "mirar", a fijarse en los detalles y a apropiarse de la obra. Se incidió en aspectos temporales con la introducción de "antes" y "ahora", para evidenciar además el grado de dominio de estos constructos. Esta actividad sirvió para propiciar una lectura de tipo sensorial, intuitiva, subjetiva y emotiva, que permitió conocer sus inquietudes y fomentar la curiosidad. Se pretendió trabajar las habilidades cognitivas que más adelante les permitirían formular hipótesis, realizar análisis y síntesis, en suma trabajar los procedimientos del método histórico, explicar de forma multicausal la sucesión, el cambio y la continuidad, para entender el entorno.

La tercera tarea, consistente en dos actividades, se planteó para abordar la lectura de tipo denotativo, lo que permitió conocer el contexto y el contenido de la obra en base al método histórico. Se trató de que decodificaran el cuadro, y visibilizaran el contexto histórico empleando la imagen, entendida ésta como vestigio de una época congelada en el tiempo. Para indagar en ella, las alumnas elaboraron un texto siguiendo la secuencia narrativa propuesta por Kieran Egan y empleando inclusores temporales recomendados por Calvani. Presentaron las principales características 
culturales del momento y a los personajes que aparecían retratados. En este texto introdujeron preguntas que facilitaron el empleo del pensamiento inferencial, inductivo y deductivo, imprescindible para elaborar hipótesis, predecir resultados y llegar a conclusiones personales y argumentadas. Además, la ubicaron en la línea del tiempo presente en el aula. En su objetivo de llenar de contenido el tiempo, diseñaron además dos actividades "las cajas de historia" y "el intruso", que permitieron a los niños identificar y explicar de forma causal los cambios y continuidades de los objetos presentes en la obra con respecto a la actualidad, así como reconocer y argumentar sobre objetos atemporales colocados en la lámina por las alumnas, y además, evaluar los aprendizajes.

La lectura de tipo formal, desarrollada en la quinta actividad, sirvió para ordenar el pensamiento visual. Se trabajó mediante dibujos de las observaciones, de las descripciones orales, y se intentó emplear todos los sentidos, evidenciando las sensaciones y los sentimientos. Identificaron entre otras cosas líneas rectas y curvas, formas planas y tridimensionales, colores, texturas y algunos cuerpos geométricos elementales, no sólo en la obra, sino también en su entorno más próximo, todo ello con el propósito de afianzar nociones espaciales, encaminadas a desarrollar su propio mapa mental.

\section{Resultados y discusión}

Con la primera actividad, en base a desplazamientos guiados, y consistente en seguir las pistas que un personaje misterioso había dejado en el aula para localizar el cuadro, se pudo evidenciar las dificultades de orientación que presentaban algunos niños. Con frases como "a la izquierda de, dos pasos enfrente de, debajo de la mesa que está a la derecha de...", tuvieron que recorrer el aula, y además representar los desplazamientos en un sencillo plano, con ayuda de la docente. Una vez localizado el cuadro, descubrieron el pergamino que les había dejado su autor, donde les planteaba una serie de preguntas que debían resolver, y que servían para organizar las tres lecturas de la obra: connotativa, denotativa y formal. La presentación del mensaje, en un papel que catalogaron de "viejo", les permitió comprender que todo lo que iban a trabajar, se había producido en un tiempo anterior, al que llamaron "hace muchos años...". Esta actividad también sirvió para evidenciar que aunque todos los niños tenían adquirida la orientación temporal referida a su tiempo personal, sin embargo confundían los tiempos verbales y los adverbios temporales cuando los transferían a situaciones nuevas. La forma de introducir el proyecto en el aula, fue muy motivadora.

La lectura connotativa, además de propiciar que se "apropiaran" de la obra empleando todos los sentidos, les ayudó a desarrollar su curiosidad. El formulario de cuestiones que las alumnas diseñaron, les permitía a los niños plantear nuevas preguntas que evidenciaran realmente sus intereses. Les intrigó fundamentalmente la indumentaria y los peinados que aparecían en la imagen, especialmente el del padre. Querían saber no sólo quiénes eran los personajes retratados, sino también por qué les habían pintado, de qué hablaban, cómo era su casa, qué comían, qué hacían en el colegio, a qué jugaban los niños... se cuestionaron acerca de los juguetes que aparecían en la pintura (el caballito de madera y el carruaje). Se les pidió que se pintaran 
dentro del cuadro y que imaginaran la conversación entre los protagonistas, para lo que se les ayudó insertando bocadillos en la figura original (Fig. 2). Acabaron la actividad escribiendo algunas palabras en una réplica de la imagen, que recibió cada uno de ellos.

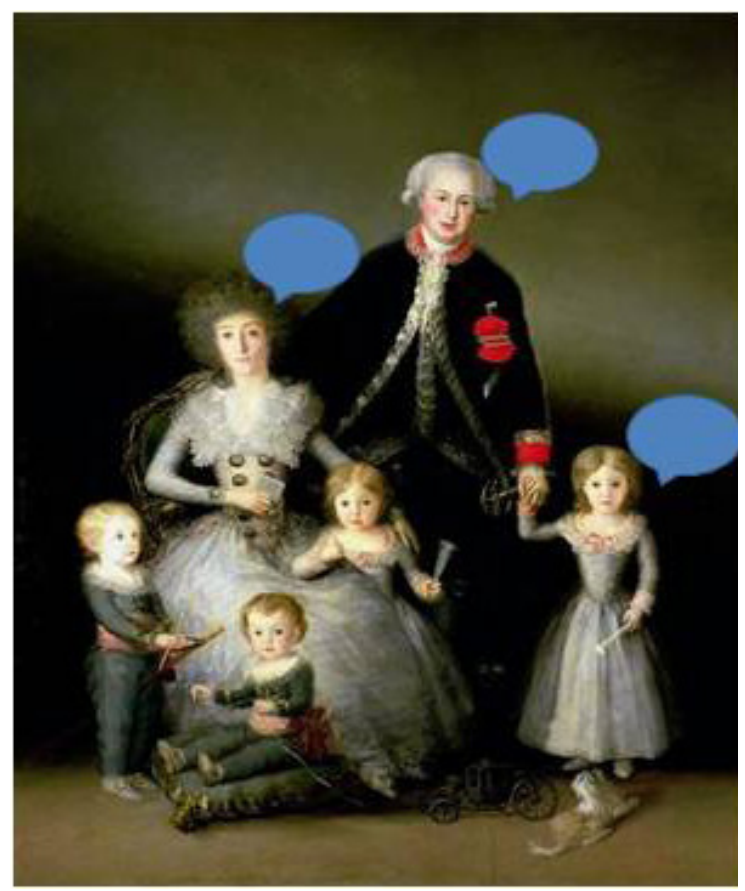

Figura 2. Imagen compuesta por la autora para realizar la lectura connotativa.

Para presentar la época, nuestras alumnas construyeron una historia dramatizada sobre contenidos culturales y sobre los personajes de la obra, siguiendo el modelo narrativo del cuento defendido por Kieran Egan (1991), en la que en base a dos contrarios, reflejaron e ilustraron el contexto de la época, y plantearon un final abierto, para que los niños enfocaran la resolución del conflicto desde diferentes puntos de vista argumentados. Finalmente, se les encargó que ordenaran secuencialmente cuatro imágenes relativas a la narración, tarea que no presentó dificultad alguna.

Con esta actividad además, se logró trabajar la empatía histórica y la narratividad, en suma las múltiples interpretaciones que tiene la historia. Ampliaron la imagen en cartón, para que los niños ocuparan el lugar de los personajes. Una vez que se "convertían" en el duque, la duquesa o sus hijos, debían actuar como ellos lo harían y explicar al resto de la clase cómo era su vida. Se puso de manifiesto que lo que se había trabajado sobre la época, había sido comprendido de forma significativa.

Estas actividades diseñadas para realizar la lectura denotativa, en base a preguntas que exigían la elaboración de hipótesis, la predicción de resultados, la búsqueda y tratamiento de información, sirvieron para trabajar contenidos culturales de la época presentes en la lámina, como el peinado, el vestido y el juguete; tomar conciencia 
de los cambios-permanencias entre el "antes" y el "ahora", y elaborar explicaciones argumentadas de forma causal (LLonch, 2010).

Con la actividad del "intruso" (Fig. 3), que sirvió además para evaluar la intervención, debían localizar el elemento extraño "perdido en el tiempo", que aparecía en la lámina, mediante la observación y la comparación, y argumentar su elección. Excepto en dos ocasiones, todos los niños tuvieron muy claro que el coche teledirigido, no debía aparecer en el cuadro, ya que "no existían los coches, sólo los carruajes de caballos", e incluso algunos argumentaron con la hipótesis de que las pilas no se "habrían inventado", lo que abrió un nuevo camino para la indagación.

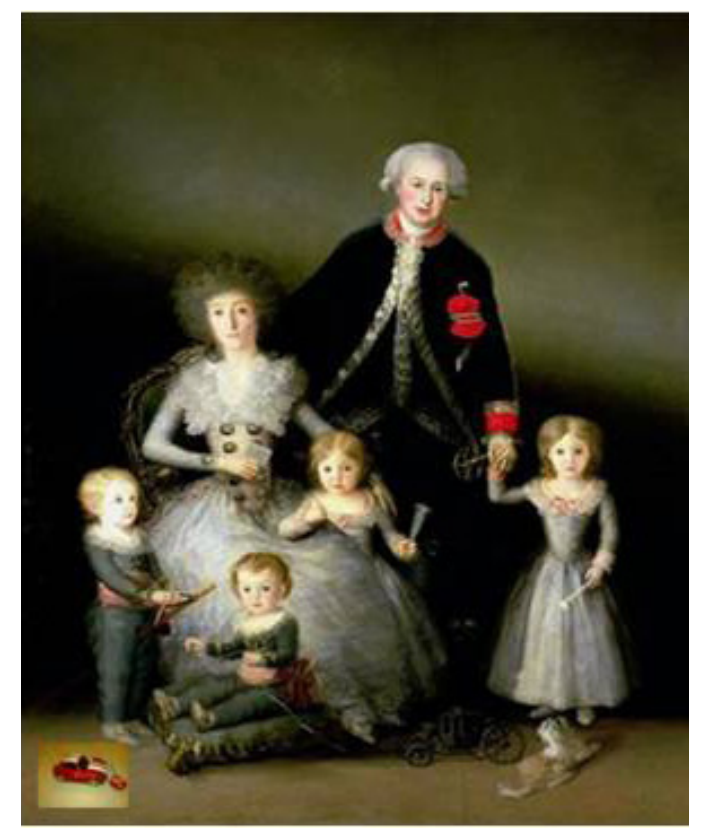

Figura 3. Imagen compuesta por la autora para realizar la lectura denotativa.

Las conversaciones entre los niños sirvieron para ampliar y consolidar el vocabulario temporal, emplearon tiempos verbales y adverbios de tiempo de forma correcta. Incluso, hubo uno que durante la actividad pidió su primer dibujo para cambiar su aspecto y adaptarlo a la moda reflejada en la lámina "con las ropas que llevaban los niños del cuadro"

Esto sirvió para incidir en el cambio experimentado por la indumentaria, consiguiendo que el resto del grupo también se replanteara la necesidad de adaptar su vestimenta a la época; y lo que es más importante, justificar este cambio desde posibles causas. Otra actividad que funcionó muy bien en el aula, fue la de las "cajas mágicas", o cajas de historia. Debían introducir en dos cajas, una con la palabra "antes" y otra con "ahora", una serie de objetos y de fotografías, entre las que se encontraban detalles del cuadro, llaves antiguas y actuales, una plancha de hierro y otra eléctrica, una fotografía de un carruaje de caballos y otra de un coche de carreras, vestidos y 
peinados de época y actuales, etc... Se trabajaron las diferencias mediante la observación y la comparación y se intentó durante toda la tarea, que argumentaran y consensuaran las decisiones. Se les ayudó con preguntas guiadas, permitiéndoles que elaboraran hipótesis y las contrastaran. Además ampliaron vocabulario y llenaron de contenido el tiempo.

Para concluir, la actividad diseñada para realizar la lectura formal de la obra, es decir para organizar el pensamiento visual, y además en este caso incidir en la orientación espacial, permitió identificar formas geométricas, comenzando por el entorno. Se les ayudó a observar lo que les rodeaba, y a geometrizar la mirada, convirtiendo los objetos próximos en triángulos, círculos, cuadrados y rectángulos, así como a reconocer líneas rectas y curvas, texturas y colores. Una vez que lo identificaron en su entorno, se les facilitó a cada uno de ellos una imagen de la obra en la que venían insertadas varias figuras geométricas que debían recortar y pegar (Fig. 4). La realización de esta actividad presentó alguna dificultad a la hora de transformar los objetos próximos en figuras geométricas, sin embargo, fueron capaces de reconocerlas en la imagen.

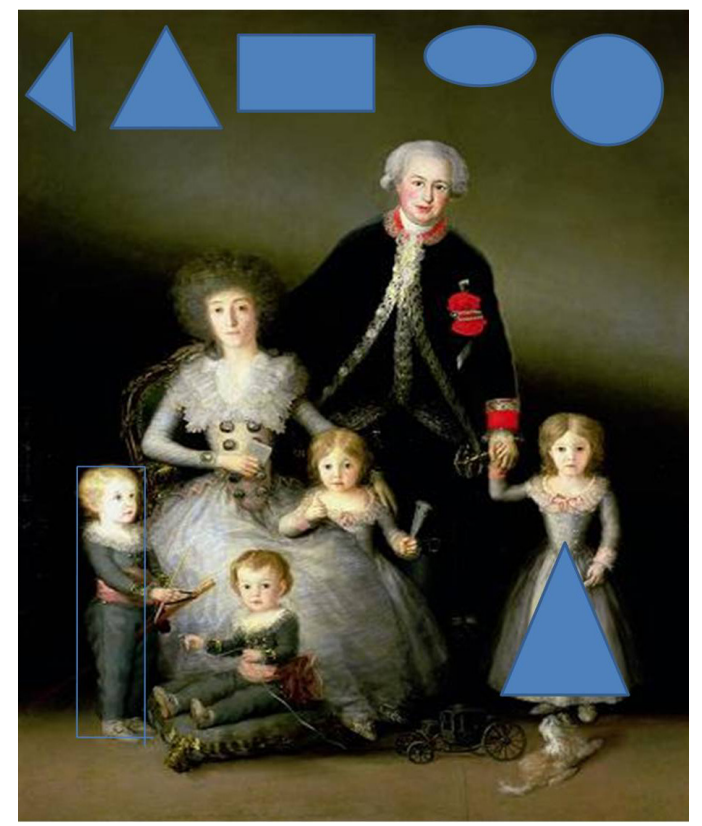

Figura 4. Imagen elaborada por la autora para realizar la lectura formal.

\section{Conclusiones}

Aunque somos conscientes de que la intervención fue corta, y que de ninguna manera se presta a conclusiones cerradas, sí podemos asegurar que este proyecto sirvió para construir un escenario de aprendizaje significativo y rico, que facilitó el desarrollo de las primeras capacidades, habilidades y destrezas necesarias para descubrir el entorno 
social en la etapa de Educación Infantil. Respondió a las expectativas de las ciencias sociales en el segundo ciclo de esta etapa, referentes a la construcción de las categorías espacio temporales propias de la misma. Se trabajó el principio de espacialidad; se introdujo el tiempo histórico en base a contenidos culturales presentes en la obra de arte, que permitieron identificar algunas señas de identidad cultural de la época y de su evolución y transformación en la actualidad. Explicar de forma causal y argumentada algunos cambios y continuidades en el modo de vida y las costumbres, todo ello a partir de la observación y la exploración de forma activa y lúdica. Además permitió trabajar la empatía histórica, la identificación con los personajes y la explicación de la historia desde diferentes puntos de vista. Valoraron la participación activa y democrática, se trabajaron las bases de la autonomía e iniciativa personal, las relaciones sociales, el respeto a las normas para afrontar la convivencia y ejercer la ciudadanía, en suma para desarrollar la competencia social. Además, de forma globalizada, se trataron conceptos propios de las matemáticas y el lenguaje, contribuyendo a desarrollar pensamiento al más alto nivel. Todo esto les permitió vivir y pensar el espacio, además llenar de sentido el tiempo, haciéndolo visualmente trasparente. Les ayudó no sólo a comprender el pasado, sino también a realizar transferencias al presente. Se sintieron seguros, autónomos y responsables de sus propias producciones, y fueron capaces de argumentar y defender sus intervenciones, interiorizando habilidades cognitivas y relacionales, desde posturas múltiples y solidarias, necesarias para la participación social de forma responsable y democrática.

Por otra parte, se produjo una clara evolución en nuestras alumnas, tanto en lo que a contenidos como a actitud se refiere, en cuanto al conocimiento artístico y su posible utilización como recurso instruccional en el proceso educativo. A pesar de que partían de conocimientos sobre historia del arte muy limitados y siempre desde planteamientos positivistas, esta experiencia les ayudó a descubrir la lectura histórica y artística que ofrece la obra de arte, y sobre todo a tomar conciencia de su poder educativo. Apreciaron su empleo como fuente de conocimiento histórico, identificando los valores y mensajes mediados por las imágenes. Aprendieron a emplearla para elaborar hipótesis, realizar análisis y síntesis, o desarrollar la abstracción y la imaginación, habilidades y estrategias que permiten sopesar diferentes versiones de una misma historia, entender las causas que la motivaron y sus efectos, así como el contexto en el que fue creada. Pusieron en valor el papel que juega el arte en el desarrollo del pensamiento, tanto a nivel afectivo y sensorial, como cognitivo. Finalmente, reconocieron a la imagen su valor para aprender a ver, vivir y pensar el espacio, así como para llenar de contenido el tiempo, también el histórico, al hacer transparentes los conceptos de sucesión, cambio y continuidad, de forma argumentada en base a explicaciones causales. Pensamos por todo ello, y teniendo en cuenta las características de nuestra sociedad, llamada de la imagen y la comunicación, que sería de gran interés formar a nuestro alumnado, tanto en conceptos básicos y en herramientas propias de la disciplina como en su didáctica, de tal forma que pueda ser competente en su empleo en el aula. Apostamos por el uso de la historia del arte para hacer transparentes los constructos espaciales y temporales, así como para introducir desde las primeras etapas educativas, contenidos y procedimientos histórico-artísticos. 


\section{Referencias}

Aranda, A. M. (2003). Didáctica del conocimiento del medio social y cultural en educación infantil. Madrid: Síntesis.

Arque I Bertrán, M. (2002). Las fuentes documentales fotográficas en la Didáctica de las Ciencias Sociales. En Carretero,A. (ed.). Primeras Jornadas Imagen, cultura y tecnología (p. 257-283). Madrid: Editorial Archiviana.

Ávila, R. M. (2003). Los maestros y los contenidos histórico-artísticos. Una experiencia de formación inicial en relación con la selección e interpretación de obras de arte para la educación primaria. Enseñanza de las ciencias sociales: revista de investigación. Barcelona, $\mathrm{n}^{\circ}$ 2, p. 93-106.

Ballesteros, E. (2003). El patrimonio y la Didáctica de las Ciencias Sociales. Cuenca: AUPDCS de profesores de Didáctica de las Ciencias Sociales AUPDCS y UCLM.

Bardavio, A. Y González, P. (2003). Objetos en el tiempo. Las fuentes materiales en la enseñanza de las Ciencias Sociales. Madrid: Akal.

Bartolomeís, F. (2006). El color de los pensamientos y de los sentimientos. Barcelona: Octaedro.

Bassedas, E., Huguet, T. y Olé, I. (2002). Aprender y enseñar en educación infantil. Barcelona: Graó.

Benites, M. y Fitchner, E. (2004). El arte como zona de desarrollo próximo para un nuevo tipo de aprendizaje. $C \& E$. Cultura y educación. Barcelona, $\mathrm{n}^{\circ} 16, \mathrm{p} .155-$ 163.

Calaf, R. y Fontal, O. (coord.). (2004). Comunicación educativa del Patrimonio referentes, modelos y ejemplos. Gijón: Trea.

Calaf Masach, R. (2010). Un modelo de investigación didáctica del patrimonio. Enseñanza de las ciencias sociales: revista de investigación. Barcelona, $\mathrm{n}^{\circ}$ 9, $\mathrm{p}$. 17-28.

Calaf, R. y Fontal, O. (2011). El conflicto en la creación artística: superar la marginalidad de la historia del arte. İber. Didáctica de las Ciencias Sociales, Geografia e Historia. Barcelona, nº 69, p. 45-54.

Candau, M. J. (2006). Percepción y didáctica del comentario de arte. Íber: Didáctica de las Ciencias Sociales, Geografia e Historia. Barcelona, no 49, p. 74-82.

Cooper, H. (2002). Didáctica de la Historia en Educación Infantil y Primaria. Madrid: Morata-MEC.

Cruz Solís, I. (2006). Arte y literatura infantil: una peculiar relación imagen-texto Revista de Didáctica Lengua y literatura. Madrid, $\mathrm{n}^{\circ}$ 18, p. 125-134.

Cuenca, J. M. y Domínguez, C. (2000). Un planteamiento socio-histórico para educación infantil. El patrimonio como fuente de trabajo para el trabajo de contenidos temporales. İber. Didáctica de las Ciencias Sociales, Geografía e Historia. Barcelona, ${ }^{\circ}$ 23, p. 113-123.

Cuenca, J. M. y Estepa, J. (2005). La caja genealógica: fuentes y tiempo en Educación Infantil. Una propuesta para trabajar con maestros. Quaderns Digitals, 37, (9). Recuperado de http://www.quadernsdigitals.net.

Cuenca, J. M. (2006). La enseñanza del Medio en Educación Infantil. Huelva: Servicio de Publicaciones de la Universidad de Huelva. 
Egan, K. (1991). La comprensión de la realidad en la Educación Infantil. Madrid: Morata.

Eisner, E. (2001): Ocho importantes condiciones para la enseñanza y el aprendizaje en las artes visuales. Arte Individuo y Sociedad. Madrid, nº13, p. 46-56.

Estepa, J., Wamba, A. M. y Jiménez, R. (2004). Fundamentos para la enseñanza y difusión del Patrimonio desde una perspectiva integradora de las Ciencias Sociales y experimentales. Investigación en la Escuela. Sevilla, no 56, p. 19-26.

Gavaldá, A. y Santisteban, A. (2003). El patrimonio fotográfico. Aportaciones a la formación universitaria y escolar. En BALLESTEROS, E. et.al. (coord.). El patrimonio y la Didáctica de las Ciencias Sociales. Cuenca: Universidad de Castilla-La Mancha.

González Monfort, N. (2008). Una investigación cualitativa y etnográfica sobre el valor educativo y el uso didáctico del patrimonio cultural. Enseñanza de las ciencias sociales: revista de investigación. Barcelona, $\mathrm{n}^{0}$ 7, p. 23-36.

Hernández Cardona, F. X. (2004). Didáctica e interpretación del patrimonio. En Calaf, R. y Fontal, O. (coords.). Comunicación educativa del patrimonio: referentes, modelos y ejemplos (p. 35-49). Gijón: Editorial Trea.

Hernández Cardona, F. (2011). La iconografía en la didáctica de las ciencias sociales. Íber: Didáctica de las Ciencias Sociales, Geografia e Historia, Barcelona, $\mathrm{n}^{\circ}$ 68, p. 7-16.

Huerta, R.(2011). Maestros, museos y artes visuales: construyendo un imaginario educativo. Arte, individuo y sociedad. Madrid, vol. 23, n 1, p. 55-72.

Juanola, R. y Calvo, M. (2006). Lo que no es nuevo puede ser actual: hacia distintos puntos de mira de la didáctica del arte. Íber: Didáctica de las Ciencias Sociales. Geografia e Historia, n $^{\circ}$ 49, p. 26-37.

Medina Ramírez, A.F. (2011). La semilla del Arte en el Arte Infantil. Arte, Individuo y Sociedad. Madrid, vol. 23, no 1, p. 81-89.

Llonch Molina, N. (2010). La indumentaria como fuente para la didáctica de la historia: problemática y estado de la cuestión. Didáctica de las ciencias experimentales y sociales. Valencia, $\mathrm{n}^{\mathrm{O}} 24$, p. 63-72.

Rielo Rodríguez, G. (2010). Un proyecto de innovación educativa: el arte y la cultura egipcia. Revista de Didácticas Especificas. Madrid, no 13, p. 93-98

Ruiz Olabuénaga, J. I. (1996). Metodología de la investigación cualitativa. Bilbao: Universidad de Deusto.

Sánchez, M. (2000). El factor espacial en el moderno concepto de la inteligencia en los procesos mentales y su relación con la expresión plástica. Arte, Individuo y Sociedad. Madrid, $n^{\circ} 12$, p. 11-16.

Socias, I. (1996). El valor del arte y la renovación de la Didáctica en las Ciencias Sociales. Íber: Didáctica de las Ciencias Sociales. Geografía e Historia. Barcelona, $\mathrm{n}^{\circ} 18$, p. 7-16.

Trepat, C. (2005). Los campos de aprendizaje y la historia del arte. Íber: Didáctica de las Ciencias Sociales. Geografía e Historia. Barcelona, $n^{\circ}$ 43, p. 44-57

Zabala, M. (2006). Reflexiones teóricas sobre patrimonio, educación y museos. Revista de Teoría y Didáctica de las Ciencias Sociales. Venezuela, nº 11, p. 233261. 
